\title{
Task Interruption and its Effects on Memory
}

\author{
Mark B. Edwards and Scott D. Gronlund \\ University of Oklahoma, USA
}

\begin{abstract}
We investigated the recovery from memory of a primary task after an interruption. If the primary task lacked associative support among its task components, recovery was more difficult following an interruption that overlapped either completely or partially in the amount of information shared with the primary task (an interruption-similarity effect). In addition, memory for completed actions was superior to memory for impending unfinished actions. However, if the primary task had associative support among its task components, there was no adverse effect of interruption similarity, and completed and unfinished actions were recalled equally well. We explore possible explanations and implications of these results.
\end{abstract}

\section{INTRODUCTION}

As any parent knows, task interruption has a marked influence on human efficiency. Bainbridge (1984) showed that malfunctions in a process control situation were often related to the occurrence of a second distracting task. For example, at Detroit Metropolitan Airport (NTSB, 1988), the flight crew were in the midst of their pre-flight checklist when they were interrupted by air traffic control prior to verifying the status of their flaps. The interruption, when coupled with other circumstances that usurped the crew's attention, resulted in the subsequent crash of the aircraft. Griffon-Fouco and Ghertman (1984) showed that the interruption of job performance accounted for better than $15 \%$ of all shut-downs of nuclear power plants. Although interruptions do not typically result in consequences as drastic as these, they do often leave the operator at a loss regarding what was being performed prior to the interruption.

Requests for reprints should be sent to Scott D. Gronlund, Department of Psychology, University of Oklahoma, Norman, OK 73019, USA. Email: sgronlund@ou.edu

Mark Edwards is now in the Human Factors Engineering group at US WEST Information Technologies, 1801 California St., Suite 1640, Denver, CO 80202, USA.

E-mail: mbedwar@uswest.com.

This worked was conducted in partial fulfilment of the $\mathrm{PhD}$ degree requirements of the University of Oklahoma by the first author under the supervision of the second author. This work was presented at the 3rd Practical Aspects of Memory conference, Washington DC, August, 1994. We thank Frank Durso for his many helpful comments on this research and this manuscript. 
Research on interruptions has a long history, beginning with the studies of Zeigarnik (1927). Zeigarnik gave her participants a series of 22 tasks, half of which were halted before completion. After all tasks had been presented, the experimenter asked the participant to name the tasks on which they had worked. Amongst those tasks that were recalled "prior to a hesitation period", an average of three more of the tasks that had been halted were recalled. Our research on interruptions differs in that our focus is on how people use their memory to recover from an interruption. Zeigarnik did not ask her participants to remember where they were in the primary task after completing the interruption nor to resume the primary task.

An improved understanding of how people use their memory to recover from interruptions may improve workplace safety and efficiency through improved design of task environments. A well designed task environment might allow the operator to develop a memory representation for the primary task that would be relatively immune to the adverse effects of interruptions. A well designed task environment could schedule unavoidable interruptions so as to be less disruptive to primary task performance. Finally, because some actions may be more prone to the disruptive effect of an interruption than others, additional cognitive and decision aids could be tailored to support the kind of information most likely to be affected by an interruption.

We explored the impact of three factors on interruption recovery: the relationship between the primary task and the interruption (the interruptionsimilarity effect), the memory representation for the primary task, and how each of these factors affected memory for actions that were completed versus actions that were impending but still unfinished at the time of the interruption. We begin by exploring the adverse effect of similarity on interruption recovery.

We defined similarity in terms of information shared between the interruption and the primary task (utilising the same constructed resources, Wickens, 1984). Czerwinski, Chrisman, and Rudisill (1991) had participants monitor a system that presented data from four spacecraft systems. Performance was worse after the similar interruption (display of the previous day's data for the same four systems) than after a dissimilar interruption (crew activities' time line). In the Czerwinski et al. (1991) study, there was complete overlap between the primary task and the similar interruption (the interruption involved the same output as the primary task). In the present studies, we explored the limits of this interruption-similarity effect by determining if partial overlap in shared information was also disruptive.

We tested an explanation for the interruption-similarity effect which assumed that the completion of a similar interruption resulted in the storage of memory traces that competed with or were confused with the traces from the primary task. This explanation could be implemented in the SAM (Search of Associative Memory) model of memory (Gillund \& Shiffrin, 1984; Raaijmakers \& Shiffrin, 1980). SAM assumes that every encoded event from the primary task is stored in 
memory as an image (a unitised collection of information about the event). Memory is defined by a matrix of strengths connecting possible retrieval cues to these images. The subsequent retrieval of traces from memory is governed by a probabilistic sampling process. The strength of connection $(S)$ of the retrieval cues to a particular trace $\left(\mathrm{T}_{\mathrm{i}}\right)$, divided by those cues' strength of connection to all the traces in memory $\left(\mathrm{T}_{\mathrm{all}}\right)$, determine the probability that image $i$ will be sampled from memory-i.e. $S\left(\mathrm{~T}_{\mathrm{i}}\right) / S\left(\mathrm{~T}_{\mathrm{all}}\right)$. (A second process governs the recovery of the information encoded in the sampled image, but that process will not affect these predictions.)

As additional traces resulting from the interruption are added to the memory matrix, they increase the denominator of the sampling equation $\left[S\left(\mathrm{~T}_{\mathrm{all}}\right)\right.$ would increase], which reduces the probability of sampling primary task traces from memory. In the memory literature, the reduced probability of recalling a given item as the number of items in memory increases is called the list-length effect (Strong, 1912). Furthermore, if the additional traces from the interruption are also strongly related to the primary task (as would result from a similar interruption), $S\left(\mathrm{~T}_{\mathrm{all}}\right)$ would increase even more, further reducing the likelihood of sampling primary task traces from memory.

A second factor we explored was how the memory representation that supported the primary task might be affected by interruption similarity. A well organised primary task representation might allow improved interruption recovery whereas an unorganised memory representation may seriously compromise interruption recovery. Two theoretical frameworks and some empirical work support this contention. Hayes-Roth (1977) proposed that changes occurred to a memory representation as task familiarity increased. She developed the Knowledge-assembly theory to describe these changes. According to Hayes-Roth (1977), learning begins with the establishment of memory representations for the elementary components of a task. With additional experience, these elementary representations become stronger and associative connections are established among them. Ultimately, a configuration can be strengthened to the point of unitisation.

A related framework was proposed by Rasmussen $(1983,1986)$. His SRK model consisted of skill-based, rule-based, and knowledge-based modes of control. The skill-based mode comprised highly organised and well-practised activity. The rule-based level included actions dependent on explicit knowledge and rules that could be verbally reported. The knowledge-based level was necessary to cope with unfamiliar situations where previously stored rules were inadequate. Bullemer and Nissen (1990) demonstrated a progression from a representation that contained individual components to one that contained relational associations, by training participants using either a random or structured sequence of light flashes. Early in training, random and structured sequences were recalled equally well, suggesting that the individual components had been established. After additional training, participants given the structured 
sequence could accurately generate the next item in the sequence, but the participants given the random sequence could not. The memory representation of the structured group apparently contained associative connections that the random group did not. Thus, learning a structured sequence resulted in an eventual change to the memory representation that led to a change in performance.

What effect will an interruption have on a primary task consisting of a fixed order of associated components? The associative connections among the components may enable the retrieval from long-term memory of the information necessary to recover from the interruption and reinstate the primary task. Furthermore, the strongly encoded primary task traces resulting from a primary task consisting of a fixed order of components would lessen the impact of the interruption. However, if the order of task components are permuted across learning trials, no associative connections should develop among components and interruption recovery may be hampered. According to SAM, an organised primary task would be instantiated by greater memory strengths or as better retrieval cues $\left[\mathrm{S}\left(\mathrm{T}_{\mathrm{i}}\right)_{\text {FIXED }}>\mathrm{S}\left(\mathrm{T}_{\mathrm{i}}\right)_{\mathrm{R} \text { ANDOM }}\right]$, which would make fixed primary task traces more likely to be recalled than permuted ones. In addition, the lack of organisation among task components may necessitate repeated re-samplings from memory to reconstruct the primary task.

The third factor affecting interruption recovery that we explored was the joint effect of memory representation and interruption similarity on memory for primary task actions that were completed versus impending but unfinished at the time of the interruption. This distinction is similar to one made in the memory literature between retrospective and prospective memory (for a review see Brandimonte, Einstein, \& McDaniel, 1996). According to Johnson and Raye (1981), the memory representation for an unfinished task (an internal memory) differs from that of a completed task (an external memory). The former consists of only cognitive operations (e.g. imaging, decision processes), the latter also contains sensory and contextual attributes that would provide more routes to retrieval that should benefit subsequent recall.

We expected that a fixed order of primary task components would result in associative connections among components that might facilitate interruption recovery. We also expected to find an interruption-similarity deficit for the group with no associative connections among task components, and expected that that deficit would be reduced when associative connections among task components were present. Finally, we expected that memory for completed actions would be superior to memory for unfinished actions. 


\section{EXPERIMENT 1}

\section{Method}

Participants. A total of 128 students from the Introductory Psychology pool at the University of Oklahoma participated in partial fulfilment of a course requirement.

Materials. We used a map of a town consisting of 19 locations (see Fig. 1). The 30 to-be-retrieved items were obtained from a norming study (see Appendix) in which we selected items based on how likely they were to be found at a particular location; 15 of the items were used in the primary task and 15 were used in the similar interruption.

Procedure. Participants performed a primary task similar to that used by Gillie and Broadbent (1989) in which items were retrieved from several locations within a hypothetical town. Unlike previous research (e.g. Gillie \& Broadbent, 1989), each participant experienced only one interruption (halfway through the sixth list), ensuring that the interruption was not anticipated.

On entering the laboratory, each participant was given a copy of the map of the town and informed that they would be retrieving items from the locations shown. Before beginning the first list they were shown an example list of three items and the sequence of computer commands required to move about the map and retrieve those items.

A trial began with the 10 items presented in the upper left-hand corner of the screen. These 10 items were randomly selected for each participant from the 15 used for the primary task. When participants felt that they had sufficiently

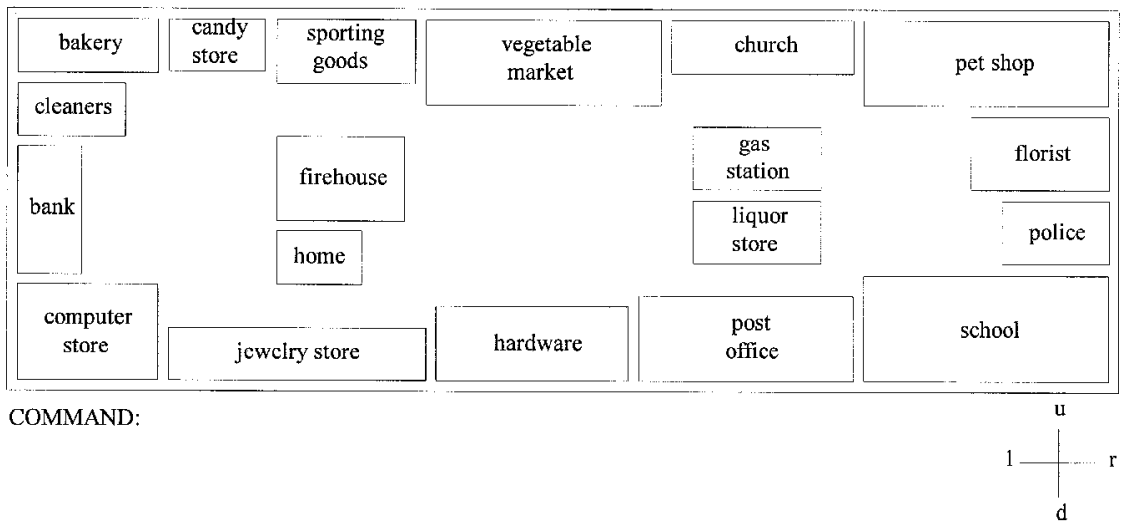

FG 1. Map of the town used in the experiments. 
memorised the items, they pressed any key to begin. The items were removed and the map appeared in the upper $\frac{3}{4}$ of the screen, remaining there as the participants retrieved the items on the list ${ }^{1}$. As participants moved through the map, they kept their current location in mind because nothing changed on the screen to signal their current location.

Participants were told that the goal of the experiment was to see how quickly and efficiently the list of items could be retrieved. They were told to minimise the number of times they looked back at the list, but not to forget an item or get one out of order. On completing the list, they were to write down, in order, the items they had retrieved.

Each participant retrieved the 10-item list six times. The participants randomly assigned to the fixed group retrieved the 10 items in the same order on each list; those in the permuted group retrieved the same items on each list, but in a different random order each time. The task was interrupted only once, after retrieving the fifth item on the sixth list.

Interruption. Immediately after retrieving the fifth item on list 6, the computer beeped and displayed a message indicating that the first phase of the experiment was over. The experimenter entered the room and reiterated that the first phase was now complete and administered either the similar or dissimilar interruption (randomly determined).

The similar interruption utilised the same locations as the primary task as well as items related to the critical items. Participants were given a sheet of paper containing the map and a new list of 15 items (those not used in the primary task), and were instructed to arrange the items so they could be retrieved most efficiently (i.e. minimise backtracking). For the dissimilar interruption, participants were given a sheet of paper with the map at the top. At the bottom of the sheet was a $5 \times 10$ matrix of two-digit integers. Participants added the numbers in each row, placing the total at the end of the row. After totalling each row, they summed the row totals to arrive at a grand total. They performed the addition on the sheet of paper. ${ }^{2}$

\footnotetext{
${ }^{1}$ The following rules applied as participants navigated through the map: (1) Home was the starting point for every trial. (2) To move, type the first letter of the desired direction of movement ( $u \mathrm{p}$, down left, right), and then the name of the location. (3) Only $90^{\circ}$ movements could be made (e.g. you could not go from the Bank to the Jewellery Store, without going through the Computer Store). (4) You had to move through adjacent buildings (e.g. you could not go directly from Home to the Liquor Store, but had to go to the Jewellery Store, Hardware Store, Post Office, and then the Liquor Store). (5) Locations could not be skipped (e.g. to get from the Jewellery Store to School, you had to go through the Hardware Store and the Post Office). (6) To retrieve an item, type $R$ and then the name of the item. (7) At any time, press $I$ to review the list for 10 seconds; the map was not visible during this time.

${ }^{2}$ We did not control the complexity of the two interruptions; however, Gillie and Broadbent (1989) found that interruption complexity did not affect the accuracy of interruption recovery.
} 
After three minutes elapsed, the computer beeped and the experimenter removed the interruption task and instructed the participant to return to the computer. When a key was pressed, the memory tests began.

Memory Tests. Participants were queried regarding the event they had just completed (completed question) and the one they were to complete next (unfinished question). We assumed that this information was central to interruption recovery. For example, if you are shopping for eggs and butter and you have just picked up eggs, that would be the completed action, and picking up butter would be the unfinished action. The completed question was: "When the computer beeped the first time, you were at a particular location; what was that location"? The unfinished question was: "When the computer beeped the first time, you had just retrieved something and were on your way to get something else; what was that something else?'. The questions were always asked in this order.

The memory tests were either recall or recognition. Participants in the recall condition received only the questions and had to generate an answer. In the recognition-unfinished condition, an alphabetised list of 15 items was provided (which included the 10 items the participants had been retrieving). In the recognition-completed condition, an alphabetised list of the 19 locations was provided. The type-of-test manipulation had no reliable effect on any of the dependent measures and will not be discussed further. Apparently, participants knew the items and locations well enough that making them available provided no added benefit.

On completion of the memory tests, participants returned to the primary task and finished retrieving the last five items from list 6. Participants began at the location that they indicated in response to the completed question. Completion of list 6 was followed by a map recall test that required reconstruction of the town by drawing and labelling each location in its appropriate spatial position on a blank sheet of paper. This task was untimed.

Design. Each participant was randomly assigned to either the fixed or permuted group. Participants completed either a similar or dissimilar interruption. The interruption was followed by a test phase involving both a completed and an unfinished question.

\section{Results and Disaussion}

Both the fixed and permuted groups were equally proficient at the primary task prior to the interruption. Both groups issued almost the same number of commands navigating through the map (about 46), and the number of commands was within two commands of optimal and did not differ between groups $[F(1$, 126) $<1, P>.10]$. In addition, participants in both groups learned the map 
equally well (ranging from 15.6 to 16.7 locations); there was no effect of group membership $[F(1,124)=1.06, P>.10]$ nor interruption type $[F(1,124)<1$, $P>$.10].

Did we succeed in inducing different memory representations in the two groups? We measured the number of items recalled at the completion of each trial. The component level of the Knowledge-assembly theory was assessed by counting the number recalled and ignoring the order in which the items were recalled (termed number recall). The associative level was assessed by counting as correct only those items recalled in order (termed order recall). One point was assigned to each word recalled in the proper sequence. If two items were recalled in proper order it counted two points, even if an intermediate item was omitted. If the ordering was switched, it counted zero points. The top panel of Fig. 2 gives the results for the fixed group and the bottom panel gives the results for the permuted group for number and order recall.

Number and order recall were analysed separately. List 6 was excluded because it included the interpolated interruption. All effects are significant at $P<.05$ unless otherwise indicated. For both number and order recall, there was a significant interaction between group (fixed or permuted) and list number [number: $F(4,121)=3.87$; order: $F(4,123)=7.15$ ]. There were significant main effects of group [number: $F(1,124)=7.5$; order: $F(1,126)=13.14$ ], with the fixed group outperforming the permuted group. There were significant main effects of list number for the fixed group [number: $F(4,121)=10.89$; order: $F(4,123)=9.26$, due to the improvement for the fixed group from list 1 to 2 for number recall $[t(63)=5.59]$, and the improvement from list 1 to 2 and 2 to 3 for order recall [minimum $t(63)=3.58$ ]. Only adjacent lists were compared. All post-hoc comparisons controlled the probability of a Type-I error by dividing $\alpha$ by the number of comparisons (Dunn, 1961).

Of primary interest was the comparison of performance on list 5, the last preinterruption list. Both groups possessed approximately equivalent componentlevel representations; everyone in the fixed group and $86 \%$ of the participants in the permuted group recalled all 10 items - although the fixed group did recall significantly more items, $t(63)=2.85$. However, the fixed group possessed a stronger representation at the associative level; number and order recall did not differ for the fixed group $t(63)=1.68, P>.05]$ but number recall was superior to order recall for the permuted group $t(63)=4.49]$.

Several other results were readily interpretable given the never-changing fixed presentation order and the ever-changing permuted one. These results provided further support for the conclusion that different memory representations underlie the task representations of the two groups. Study time (time spent studying each list prior to retrieving the items) was less for the fixed group $[F(1,126)=21.89]$ (see Fig. 3, top panel). Post-hoc comparisons showed that the fixed group spent less time studying Lists 3 through 6 . Overall, study time decreased as a function of list number $[F(5,122)=14.35]$ and the interaction was 
Fixed
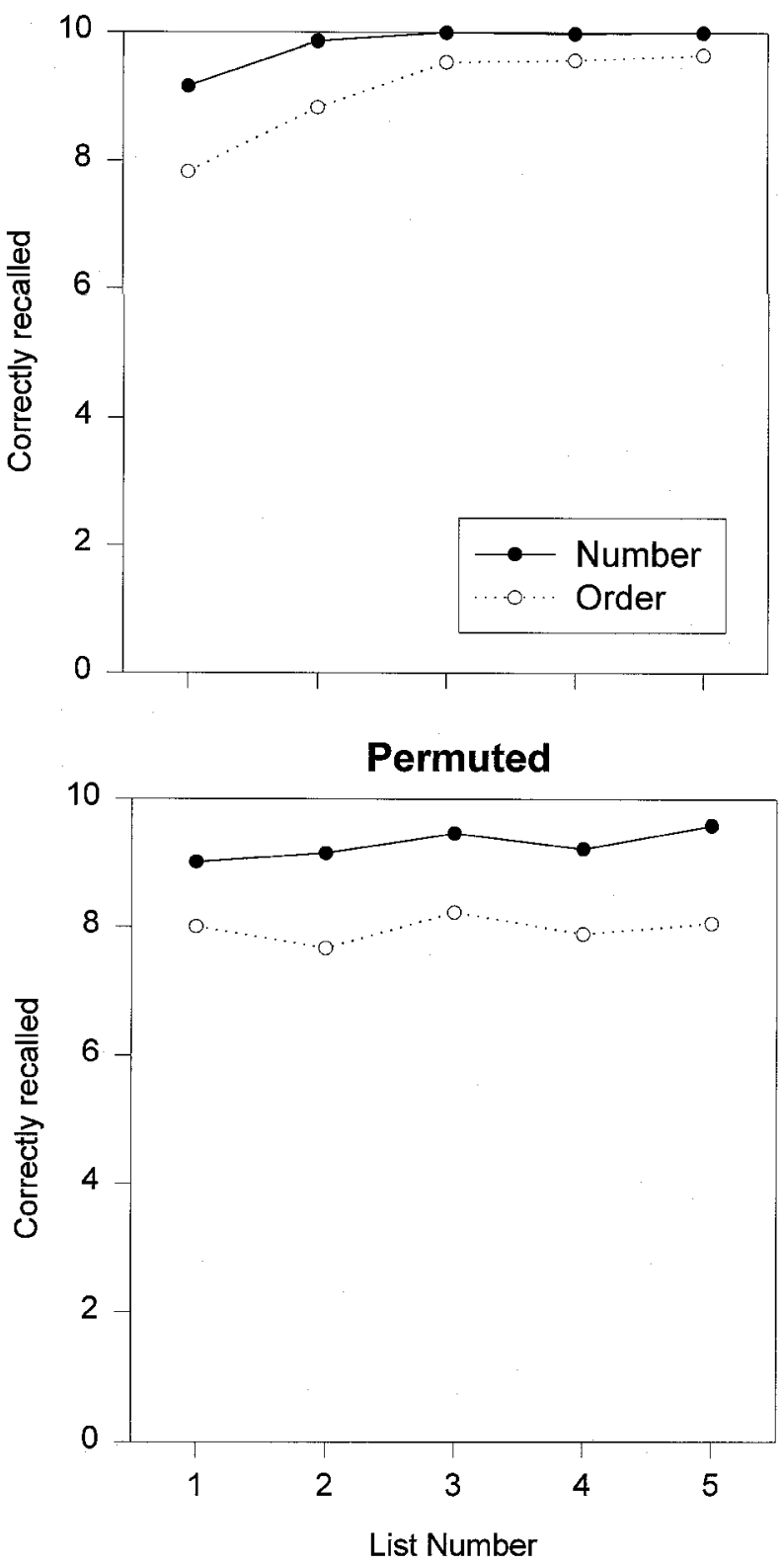

FG 2 Recall of the items at conclusion of each of the first five lists scored for number correct (number) and number correct in order (order). The top panel is for the fixed group and the bottom panel is for the permuted group. 


\section{Study Time}

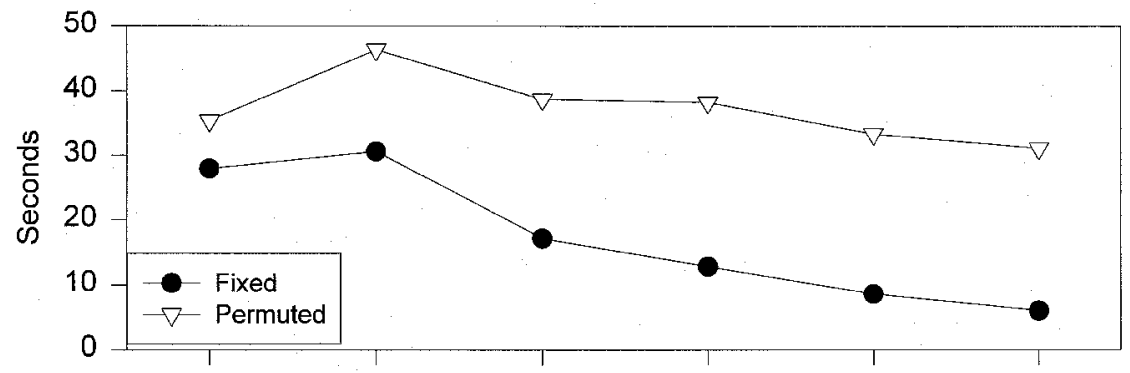

\section{List Completion Time}

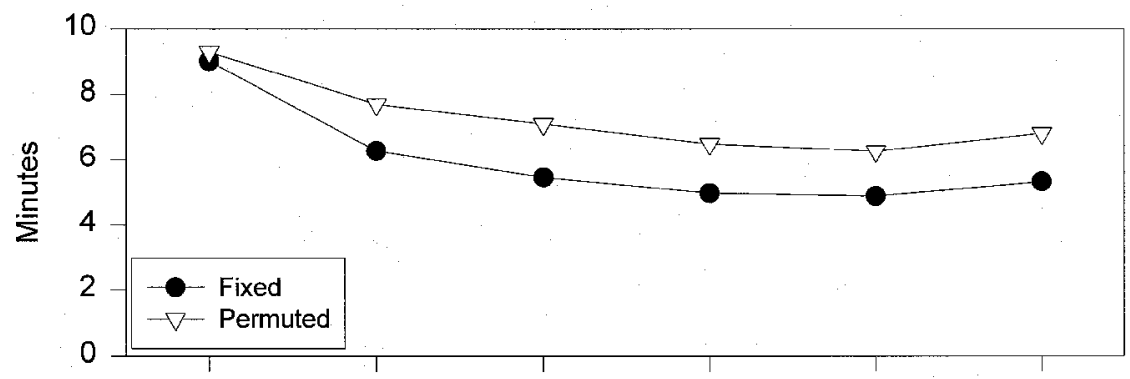

\section{Number of Looks Back at List}

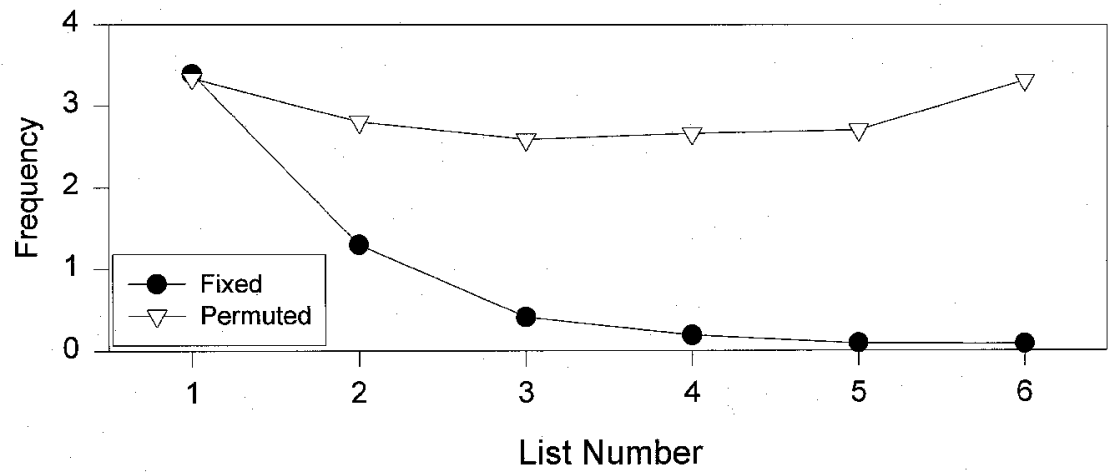

FG 3. Top panel: Amount of time spent studying a list prior to beginning the task for the fixed and permuted groups. Middle panel: Total time spent retrieving all the items on the list for the fixed and permuted groups. Time to complete the interruption is subtracted from the List 6 time. Bottom panel: Number of times participants reviewed list of items during the completion of the task. 
significant $[F(5,122)=3.66]$. The fixed group also completed retrieving all the items on a list more quickly $[F(1,126)=17.79]$ (see Fig. 3 , middle panel) ${ }^{3}$. The longer list completion times for the permuted group were due to more looks back at the list during retrieval $F(1,126)=102.6]$ (see Fig. 3, bottom panel). There was no difference between the two groups in list completion time (and number of looks) on lists 1 and 2, but the fixed group was faster (and had fewer looks back) for every list thereafter. We believe that there was sufficient evidence to support the contention that we induced different memory representations in the two groups. Did the two representations have different consequences for interruption recovery?

The accuracy of responses to the unfinished and completed questions are shown in Fig. 4. The top panel shows the results for the fixed group and the bottom panel shows the results for the permuted group. The fixed group performed better than the permuted group on unfinished and completed questions after the similar interruption $\left[\chi^{2}(1)=5.5\right.$ and $\chi^{2}(1)=7.8$, respectively]. For the dissimilar interruption, the fixed group was better only for the unfinished question $\left[\chi^{2}(1)=7.33\right]$. However, we must be careful when comparing the fixed to the permuted group. It is possible that the inclusion of a no-interruption control (i.e. primary task performance would be halted but the completed and unfinished questions would be asked immediately) would have shown that fixed group performance was better than permuted group performance even without the interruption (however, note that performance for the completed question after the dissimilar interruption was actually slightly better for the permuted group). However, this limitation does not affect conclusions about the interruption-similarity effect nor comparisons involving completed and unfinished actions because those comparisons are restricted to be within the fixed or within the permuted group rather than between the two groups.

Performance for each condition was compared to chance using a normal approximation to the binomial (Hays, 1988), controlling $\alpha$ at .006 (.05 divided by the number of tests). Chance was assumed to be 1/10th for the items and 1/ 19 th for the locations. Performance was above chance for all conditions except for the permuted group on the unfinished question after the similar interruption. It was also true that performance for the permuted group on the completed question was worse after the similar than after the dissimilar interruption $\left[\chi^{2}(1)=9.14\right]$. Taken together, these two results document the detrimental effect of the similar interruption on the permuted group.

Performance on the completed questions was better than performance for the unfinished questions, but not reliably so. However, this comparison was suspect because spatial information (the locations tapped by the completed question) is

\footnotetext{
${ }^{3}$ Completion time for list 6 was corrected by subtracting out the time to complete the interruption (three minutes) as well as the time to answer each memory question.
} 
Fixed

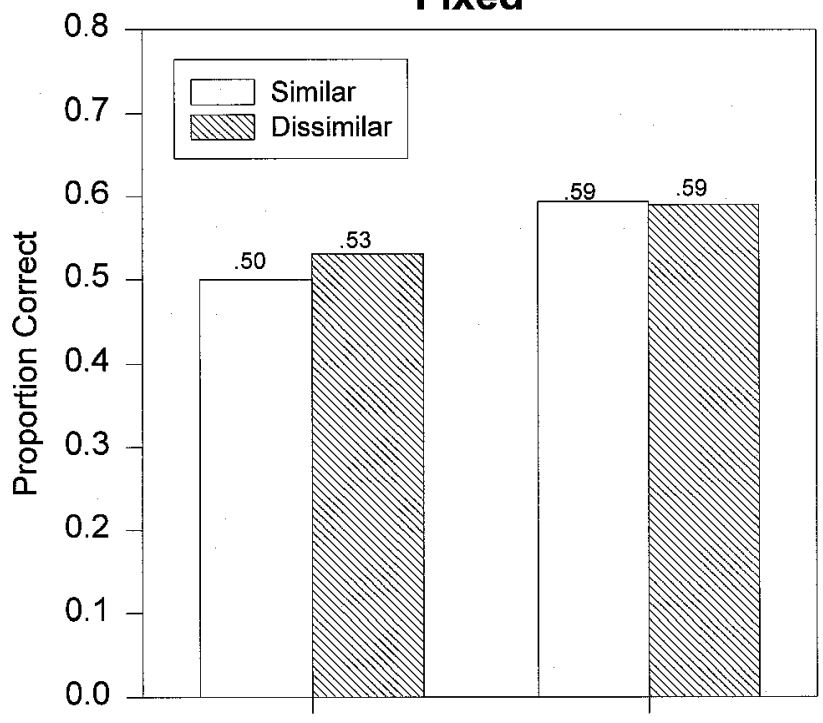

Permuted

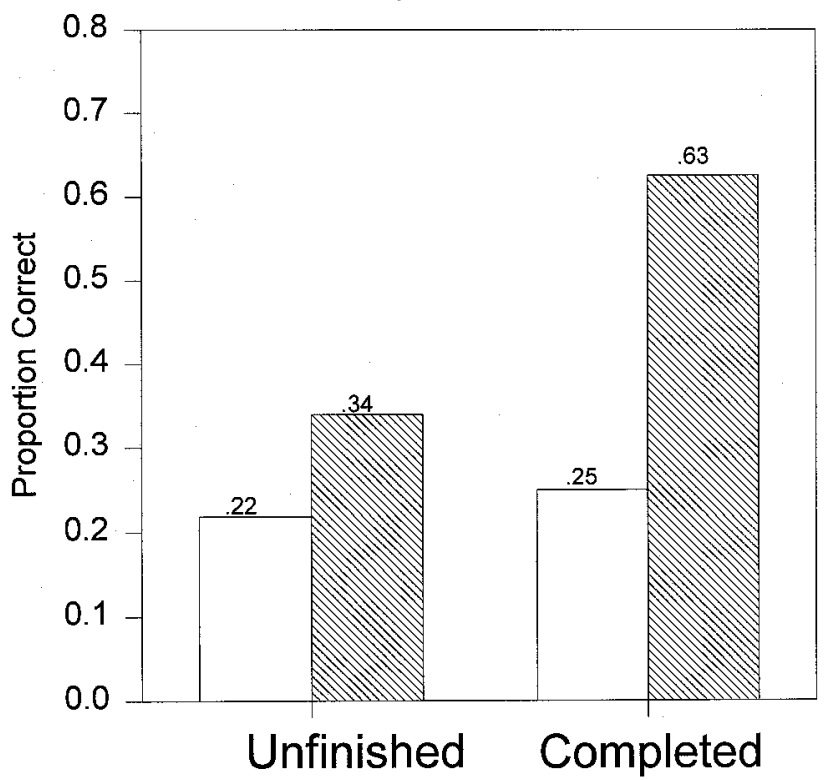

FG 4. Proportion correct for the unfinished and completed questions after the similar or dissimilar interruption. The top panel is for the fixed group and the bottom panel is for the permuted group. 
typically recalled better than verbal information (the items tapped by the unfinished question) (Paivio, 1971). This problem was rectified in Experiment 3.

By having the participants retrieve the items in a fixed order, we succeeded in creating a representation at the associative level for the fixed group. The fixed group performed better on the unfinished question, and on the completed question after a similar interruption. Participants accomplished this despite spending significantly less time studying the list before beginning to retrieve, and many fewer times reviewing the list during retrieval of the items. We replicated an interruption-similarity decrement, although only for the permuted group. Training on a task to the point that associative connections were established overcame the detrimental effect of a similar interruption. Because of our interest in determining what makes an interruption disruptive, we decided to restrict our remaining experiments to the permuted group because that was the group that was differentially affected by the type of interruption, as well as the one that showed a difference in recallability between unfinished and completed actions. We shall return to possible explanations for the lack of these differences in the fixed group in the General Discussion.

In Experiment 1, the similar interruption had encompassed both aspects of the primary task - the locations and related items - and for the permuted group it impaired memory for both. In Experiments 2 and 3, the similar interruption will involve only the items. Does an interruption involving the items harm memory only for the items and not for the locations?

\section{EXPERIMENT 2}

\section{Method}

Participants. A total of 66 students from the Introductory Psychology pool at the University of Oklahoma participated in partial fulfilment of a course requirement.

Procedure. The procedure was identical to that of Experiment 1 except for the following changes. First, only the permuted group was included. Second, the similar interruption now required participants to unscramble a list of 15 anagrams. They were told that some of the anagrams were items that they had been retrieving (in fact 10 of them were). Third, the order in which the memory questions were asked was randomised. Fourth, all questions required the recall of the requested information.

\section{Results and Disaussion}

The number of commands did not differ from optimal efficiency $[F(5,60)=.78$, $P>$.10]. Memory for the spatial arrangement of the map did not differ with interruption type $[t(64)=.07, P>.10]$, and the number of locations recalled was 
close to what was observed in Experiment $1^{4}$. The recall results closely replicated the permuted group in Experiment 1; number recall was superior to order recall (see top panel of Fig. 5). On list 5, for example, number recall was greater than order recall $[M=9.4$ vs. $8.5, t(65)=2.6]$.

Figure 6 gives the proportion correct on the two memory questions (top panel, Experiment 2). We again see evidence of an interruption-similarity effect. Performance was compared to chance using a normal approximation to the binomial (Hays, 1988). Performance was no better than chance for the unfinished question after the similar interruption. For the completed question, performance after the similar interruption was significantly worse than after the dissimilar interruption, $\chi^{2}(1)=6.11$. This was the same pattern that we observed for the permuted group in Experiment 1. Furthermore, the interruption-similarity effect found in Experiment 1 does not appear to be highly circumscribed. In Experiment 2, memory for location (completed question) was harmed comparably to what was observed in Experiment 1, despite the fact that the similar interruption in Experiment 2 involved only the items.

\section{EXPERIMENT 3}

In Experiment 2, the unfinished question asked about the item to be retrieved next and the completed question asked about the location just visited. In Experiment 3, both the completed and unfinished questions were item-based (the item that they had just retrieved and the item they were to retrieve next, respectively). This allowed memory for completed and unfinished actions to be compared directly without confounding verbal and spatial information.

\section{Method}

Participants. A total of 60 students from the Introductory Psychology pool at the University of Oklahoma participated in partial fulfilment of a course requirement.

Procedure. The procedure was identical to that of Experiment 2 except that both the completed and unfinished questions involved the items.

\footnotetext{
${ }^{4}$ Study time, list completion time, and number of looks back at the list closely replicated the permuted group in Experiment 1. Study time showed no effect of list number $(F(5,60)=1.16$, $P>.10]$. List completion time varied as a function of list number $[F(5,58)=13.80]$. Post-hoc comparisons showed a significant decrease from list 2 to 3 and 3 to 4 , and an increase from list 5 to 6 . The number of looks changed as a function of list number $[F(5,60)=9.02]$. Post-hoc tests showed a decrease from list 1 to 2 and 3 to 4, and an increase from list 5 to 6 , due in large part to an increased number of post-interr uption looks -2.8 post-interr uption vs. 1.0 pre-interr uption looks, $t(64)=12.6$.
} 


\section{Experiment 2}

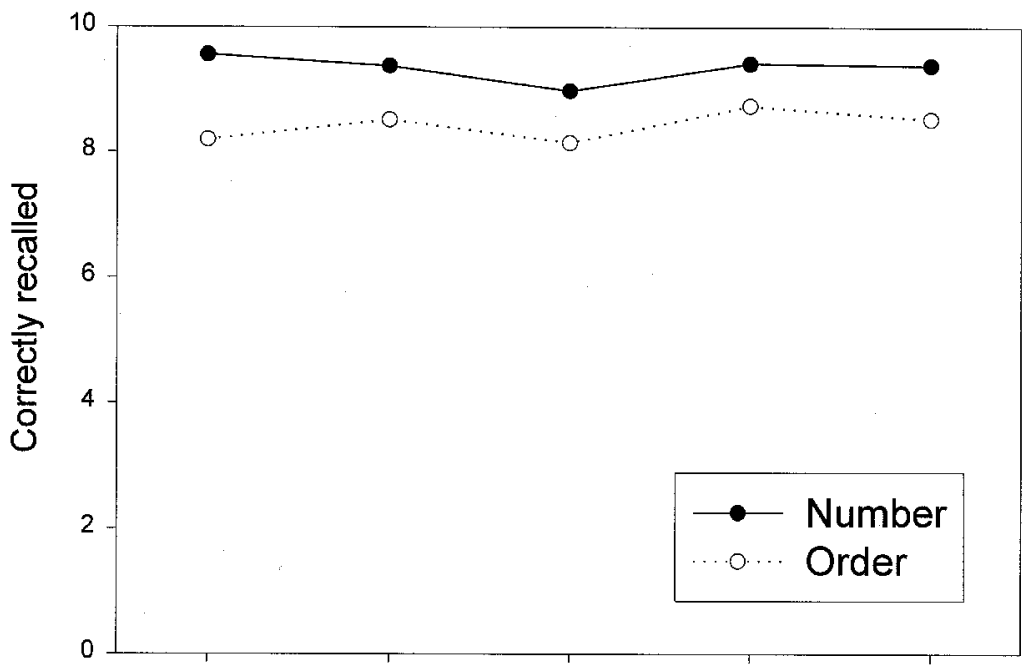

\section{Experiment 3}

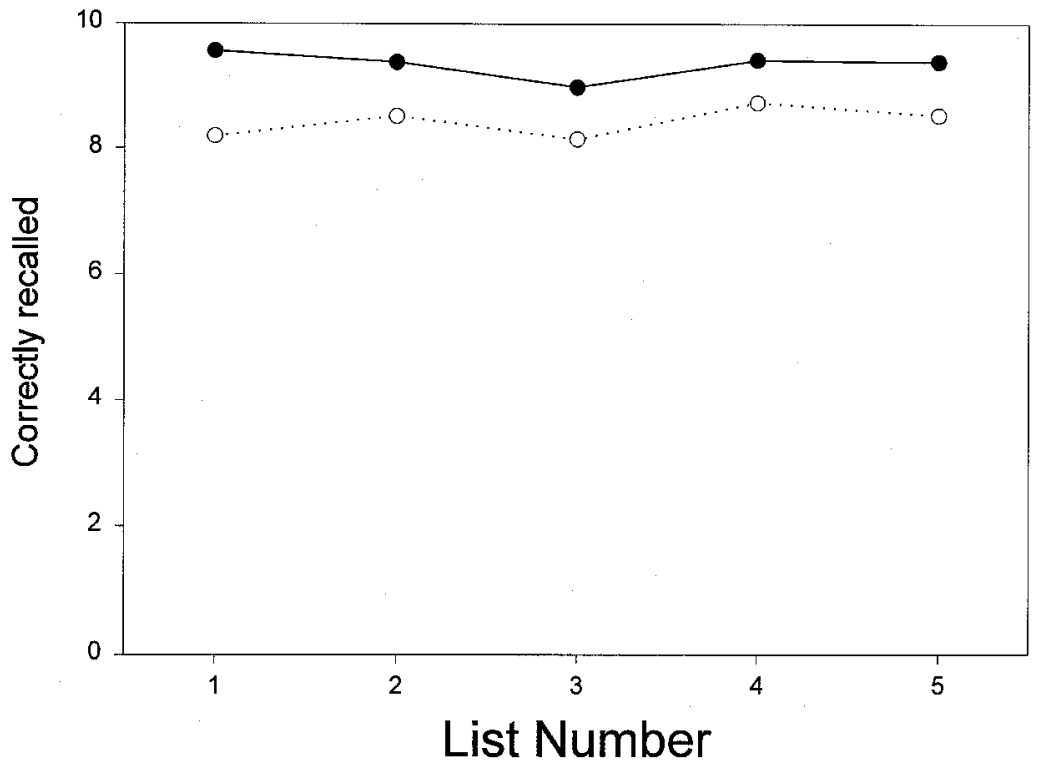

AG 5. Number and order recall for Experiment 2 (top panel) and Experiment 3 (bottom panel). 


\section{Results and Discussion}

The number of commands did not differ from optimal efficiency $[F(5,54)<1$, $P>$.10]. Memory for the spatial arrangement of the map did not differ with interruption type $[t(58)=.47, P>.10]$, and the number of locations recalled was close to what was observed in Experiment $1^{5}$. The recall results closely replicated the permuted group in Experiments 1 and 2; number recall was superior to order recall (see bottom panel of Fig. 5). On list 5, for example, number recall was greater than order recall $[M=9.2$ vs. $7.95, t(59)=3.4]$.

Figure 6 gives the proportion correct on the two memory questions (bottom panel, Experiment 3). We again see evidence of an interruption-similarity effect. Performance was compared to chance using a normal approximation to the binomial (Hays, 1988). Performance was no better than chance for the unfinished question after the similar interruption. For the completed question, performance after the similar interruption was worse than after the dissimilar interruption, although the effect was only marginal $\chi^{2}(1)=3.3, P<.07$. This was the same pattern that we observed for the permuted group in Experiments 1 and 2.

Performance after the dissimilar interruption was also not different from chance for the unfinished question - although it was marginally better than after the similar interruption, $\chi^{2}(1)=3.3, P<.07$. The fact that performance for the completed question was above chance after both interruptions provides some statistical support for a trend prominent in Experiments 1 and 2; performance on the completed questions was superior to performance on the unfinished questions.

\section{GNRALDISCUSSION}

We explored the interruption-similarity effect by varying the degree of overlap between a primary task and a similar interruption, and by examining primary tasks with or without associative support. In Experiment 1, the overlap between the primary task and the interruption was nearly complete for the similar interruption (involving the same locations and related items), but the task representation engendered by the fixed order of training provided complete resistance to the similar interruption, contrary to the qualitative predictions extracted from the SAM model. The permuted group, however, was adversely affected by the similar interruption. In Experiment 2, we showed that the degree

\footnotetext{
${ }^{5}$ Study time, list completion time, and number of looks back at the list closely replicated the permuted group in Experiments 1 and 2. Study time showed no effect of list number $[F(5,54)<1$, $P>$.10]. List completion time varied as a function of list number $[F(5,54)=4.9]$. Post-hoc comparisons showed a significant decrease from list 1 to 2 and an increase from list 5 to 6 . The number of looks changed as a function of list number $[F(5,54)=2.55]$. Post-hoc tests showed an increase from list 5 to 6 , due in large part to an increased number of post-interruption looks- $(2.5$ post-interr uption vs. 1.0 pre-interr uption looks, $t(58)=9.1]$.
} 


\section{Experiment 2}

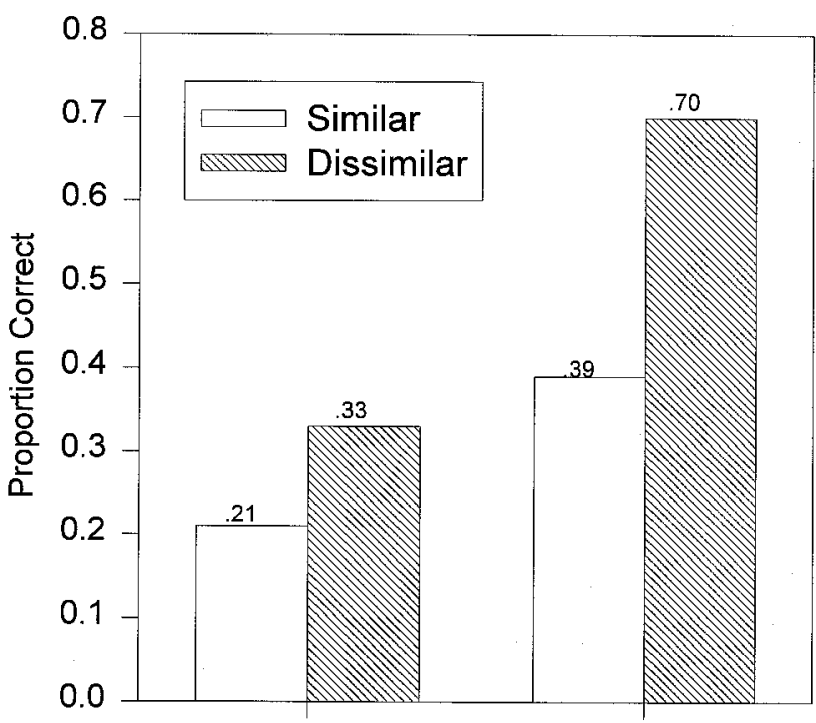

\section{Experiment 3}

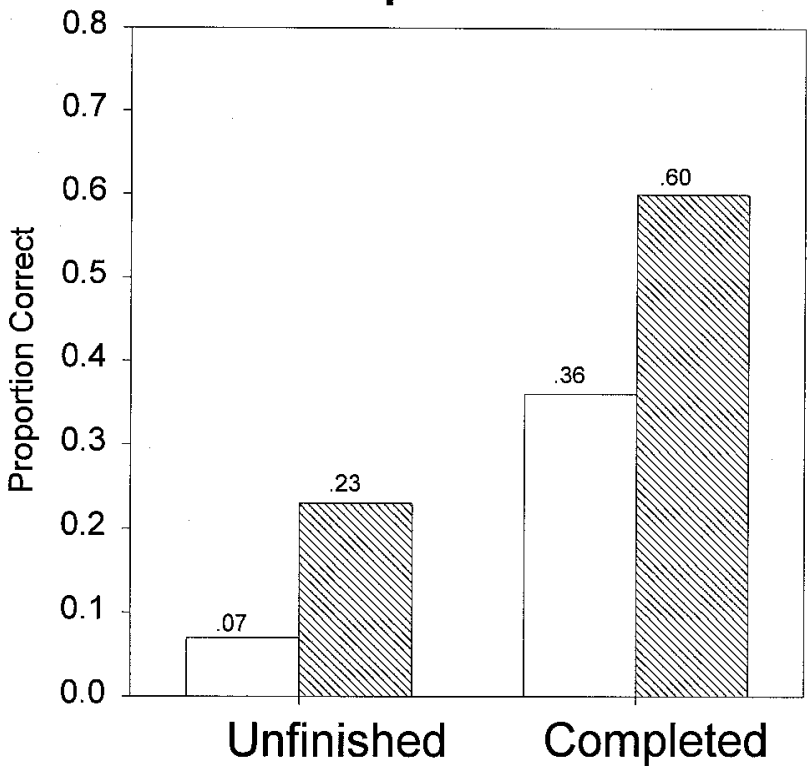

FG 6. Proportion correct for the unfinished and completed questions after the similar or dissimilar interruption. Experiment 2 is shown in the top panel and Experiment 3 is shown in the bottom panel. 
of overlap for a similar interruption need not be complete to be disruptive; information regarding location was no more adversely affected by an interruption that utilised location information (Experiment 1) than one that focused solely on the items (Experiments 2 and 3).

We also examined what impact the representation of the primary task and interruption similarity had on memory for completed and unfinished actions. Completed and unfinished actions were equally well remembered by the fixed group, but there was a consistent trend showing superior memory for completed actions over unfinished actions for the permuted group (see especially Experiment 3). We next explore some possible explanations and potential implications of each of these findings.

The fixed group developed a representation at the associative level (HayesRoth, 1977), which resulted in resistance to the effects of a similar interruption and equivalent recall between completed and unfinished actions. Perhaps the associative interconnections supported recovery of the entire task structure for the fixed group rather than only fragments of it. This idea is related to a theoretical framework proposed by Ericsson and Kintsch (1995). Ericsson and Kintsch argued that a construct called long-term working memory (LT-WM) developed as a result of the repeated storage and retrieval of task-relevant information (over a much longer period of time than was used here). Long-term working memory is a retrieval structure which consists of pointers held in working memory that point to permanent information in long-term memory (LTM). According to Ericsson and Kintsch (1995, p. 212), "storage of information in LT-WM implies that most types of accessible information in working memory will remain in LTM during an interruption of skilled activities and that access to them can be easily reinstated by reactivation of necessary retrieval cues.' We think that the representation developed by the fixed group is analogous to (although not as well developed as) the memory representation that a skilled operator develops for a task, assuming that there is sufficient consistent repetition of actions within the task.

The lack of an effect of the similar interruption on performance for the fixed group was contrary to what was predicted by the SAM model. Stronger encoding of the primary task should have lessened, but not eliminated, the similar interruption decrement. The lack of an interruption-similarity effect for the fixed group is consistent with related findings in the memory literature. For example, Hockley (1991) found that memory for associations between randomly paired words was more resistant to the interfering effects of intervening events than was memory for individual words (which would be at Hayes-Roth's individualcomponent level). Hockley attributed this lack of forgetting to the distinctiveness of associations relative to individual words.

The SAM model could predict a similar-interruption deficit like that observed for the permuted group; however, the model would have expected the size of the effect to be lessened in Experiments 2 and 3 when the interruption traces only 
partially overlapped with the primary task traces. This did not appear to be the case, although this was a cross-experiment comparison and a more powerful comparison could demonstrate such a decline in the size of the interruptionsimilarity effect. Furthermore, it is possible that the items and the locations were so strongly interrelated in this task that FLORIST came to mind once OSER was unscrambled. If this was true, the Experiment 2 and 3 interruptions were no less similar to the primary task than was the Experiment 1 interruption.

These experiments do not address the issue of whether the interruptionsimilarity effect requires the laying down of similar traces or whether an interruption that laid down dissimilar traces might also hinder interruption recovery. In the present experiments, it was probably the case that no traces were stored as a result of the dissimilar interruption; only working memory was necessary to complete the addition task. Although the SAM model would predict that similar interruption traces should be more disruptive, dissimilar traces would also hinder interruption recovery (the list-length effect).

Interruption similarity can also be defined in terms of shared processes or base resources (Wickens, 1984). For example, Gillie and Broadbent (1989) found that the free recall of items from a primary task was slowed, and accuracy decreased, by an interruption that also required free recall. It was possible that the similar interruption and the primary task in the present experiments shared more processes than did the dissimilar interruption and the primary task. This could be another reason why the similar interruption was more detrimental to performance.

The third principal finding of these studies involved the trend towards better memory for completed over unfinished actions. This finding, if replicated, would be consistent with the predictions of Johnson and Raye (1981). Interestingly, if the primary task was supported by associative connections among task components, no such advantage for completed actions was observed. This was probably the result of the item-to-item links in the fixed group, which might be sufficient to overcome the relatively more impoverished encoding of the unfinished actions.

We think that an improved understanding of the effect of interruptions on memory could improve the design of various task environments. A well designed task environment might allow for the creation of associative connections among task components that would result in a mental representation for the task that was relatively immune to the adverse effects of interruptions. With sufficient training, an associative representation may even be transformed into a unitised representation (Hayes-Roth's, 1977, third level), although it is unclear how interruptions would affect a primary task organised in this way. On the one hand, an interruption may not be disruptive at all because the primary task can be accomplished with so few resources that the interruption could essentially be completed in parallel with it. On the other hand, the primary task may be so well learned that it was not being monitored by the operator. On 
completion of the interruption, the operator may have to start the primary task over again, having no idea where to resume.

If a primary task could not be redesigned to allow for the development of associative connections, it would be better to schedule unavoidable interruptions so that they do not occur in conjunction with similar primary task activities, thereby limiting their disruptive effect. Perhaps they could be delayed until a more opportune time, or responsibility for completing the interrupting activity could be passed to another operator in the system. Finally, if the task environment does not support the creation of associative connections among task components, cognitive aids that maintained unfinished actions would benefit performance. This could be something as simple as a piece of scratch paper, or as sophisticated as a personal digital assistant or elaborate computer interface. For example, in the air traffic control domain, controllers offset flight progress strips (paper strips that contain the pilot's flight plan) as reminders to take future actions (Vortac, Edwards, Fuller, \& Manning, 1993).

There is a burgeoning accumulation of knowledge examining how people interact with complex environments and complex systems (e.g. see overviews by Adams, Tenny, \& Pew, 1991; Meyer \& Kieras, 1997). Adams et al. called it a paradigm shift for psychology. We believe that an examination of the cognitive management of interruptions has much to contribute to an increased understanding of cognition in these environments.

Manuscript received 7 March 1997

Manuscript accepted 23 June 1997

\section{REERENES}

Adams, M.J., Tenny, Y.J., \& Pew, R.W. (1991). State-of-the-art report: Strategic workload and the cognitive management of advanced multi-task systems (CSERIAC 91-6). Wright-Patterson Air Force Base, OH: Crew System Ergonomics Information Analysis Center.

Bainbridge, L. (1984). Diagnostic skills in process operation. Proceedings of 1984 International Conference on Occupational Ergonomics, 2, 1-10.

Brandimonte, M., Einstein, G.O., \& McDaniel, M.A. (Eds.) (1996). Prospective memory: Theory and applications. Hillsdale, NJ: Lawrence Erlbaum Associates Inc.

Bullemer, P., \& Nissen, M.J. (1990). Attentional orienting in the expression of procedural knowledge. Presented at the 31st Annual Meeting of the Psychonomic Society, New Orleans, LA.

Czerwinski, M.P., Chrisman, S.E., \& Rudisill, M. (1991). Interruptions in multitasking situations: The effect of similarity and warning (technical report JSC-24757). Houston, Tx: National Aeronautics and Space Administration.

Dunn, O.J. (1961). Multiple comparisons among means. Journal of the American Statistical Association, 56, 52-64.

Ericsson, K.A., \& Kintsch, W. (1995). Long-term working memory. Psychological Review, 102, 211-245.

Gillie, T., \& Broadbent, D. (1989). What makes interruptions disruptive? A study of length, similarity, and complexity. Psychological Research, 50, 243-250.

Gillund, G., \& Shiffrin, R.M. (1984). A retrieval model for both recognition and recall. Psychological Review, 91, 1-67. 
Griffon-Fouco, M., \& Ghertman, F. (1984). Recueil de données sur les facteurs humains à Electricité de France, Operational Safety of Nuclear Power Plants. Vienna International Atomic Energy Agency, 157-172.

Hayes-Roth, B. (1977). Evolution of cognitive structures and processes. Psychological Review, 84, 260-278.

Hays, W.L. (1988). Statistics. Orlando, FL: Holt, Rinehart, \& Winston.

Hockley, W.E. (1991). Recognition memory for item and associative information: A comparison of forgetting rates. In W.E. Hockley \& S. Lewandowsky (Eds.), Relating theory and data: Essays on human memory in honor of Bennet B. Murdock (pp.227-248). Hillsdale, NJ: Lawrence Erlbaum Associates Inc.

Johnson, M.K., \& raye, C.L. (1981). Reality monitoring. Psychological Review, 88, 67-85.

Meyer, D.E., \& Kieras, D.E. (1977). A computational theory of executive processes and multipletask performance: Part 1. Basic mechanisms, Psychological Review, 104, 3-65.

National Traffic Safety Board (1988). Aircraft accident report-Northwest Airlines, McDonnell Douglas DC-9-82, N312RC, Detroit Metropolitan Wayne Co. Airport. Washington, DC: NTSB.

Paivio, A. (1971). Imagery and verbal processes. New York: Holt, Rinehart, \& Winston.

Raaijmakers, J.G.W., \& Shiffrin, R.M. (1980). SAM: A theory of probabilistic search of associative memory. In G.H. Bower (Ed.), The psychology of learning and motivation (Vol. 14)., New York: Academic Press.

Rasmussen, J. (1983). Skills, rules, and knowledge; signals, signs, and symbols, and other distinctions in human performance models. IEEE Transactions on Systems, Man, and Cybernetics, 3, 257-266.

Rasmussen, J. (1986). Information processing and human-machine interaction. New York: Elsevier Science Publishing.

Strong, E.K. Jr. (1912). The effect of length of series upon recognition memory. Psychological Review, 19, 447-462.

Vortac, O.U., Edwards, M.B., Fuller, D.K., \& Manning, C.A. (1993). Automation and cognition in air traffic control: An empirical investigation. Applied Cognitive Psychology, 7, 631-651.

Wickens, C.D. (1984). Processing resources in attention. In R. Parasuraman \& D.R. Davies (Eds.), Varieties of attention. Orlando, FL: Academic Press.

Zeigarnik, B. (1927). Das behalten erledigter und underledigter handlungen. Psychologische Forschung, 9, 1-85. 


\section{APPENIX}

A norming study was conducted to select locations and items. We wanted a set of items that could only be retrieved at one of the possible locations.

\section{Method}

Participants. Participants were 150 students from the Introductory Psychology pool at the University of Oklahoma who participated in partial fulfilment of a course requirement.

Design. A list of 22 locations was generated, together with three or four items likely to be found at each location. For example, the items at the Computer Store included a monitor, floppy disks, a modem, and a keyboard. A sheet was prepared with the locations on the left and the items on the right, and was distributed to a classroom of students who were instructed to place each item by the location that first came to mind. Each item was to be listed beside a location, but not all locations needed items.

\section{Results}

Three locations were eliminated (Car Dealer, Beauty Shop, Restaurant) because there was substantial disagreement over the items placed at these and at least one other location (e.g. motor oil could be found at either the Gas Station or the Car Dealer). Of the remaining 19 locations, 15 were selected because they contained two highly probable items. One of these items was used in the main task, the other was used for the similar interruption. Four additional locations served as fillers; none of the items could be retrieved from these locations (Home, Church, Police Station, and Firehouse). The locations, items, and likelihood that each item was paired with a location, are given in Table A1. 


\section{TABEA1}

First and Second Mbst Frequently Listed Items for each Location

\begin{tabular}{|c|c|c|}
\hline Location & Item & Likelihood \\
\hline \multirow[t]{2}{*}{ Bakery } & bagel & $100 \%$ \\
\hline & bread & $98 \%$ \\
\hline \multirow[t]{2}{*}{ Candy Store } & taffy & $99 \%$ \\
\hline & jaw breakers & $100 \%$ \\
\hline \multirow[t]{2}{*}{ Sporting Goods } & sweatshirt & $95 \%$ \\
\hline & football & $98 \%$ \\
\hline \multirow[t]{2}{*}{ Vegetable Market } & broccoli & $98 \%$ \\
\hline & cauliflower & $97 \%$ \\
\hline \multirow[t]{2}{*}{ Pet Shop } & $\operatorname{dog}$ & $95 \%$ \\
\hline & cat & $97 \%$ \\
\hline \multirow[t]{2}{*}{ Florist } & roses & $99 \%$ \\
\hline & tulips & $100 \%$ \\
\hline \multirow[t]{2}{*}{ School } & grades & $100 \%$ \\
\hline & children & $73 \%$ \\
\hline \multirow[t]{2}{*}{ Post Office } & stamps & $99 \%$ \\
\hline & mail & $100 \%$ \\
\hline \multirow[t]{2}{*}{ Jewellery Store } & ring & $98 \%$ \\
\hline & necklace & $98 \%$ \\
\hline \multirow[t]{2}{*}{ Computer Store } & floppy disks & $100 \%$ \\
\hline & modem & $100 \%$ \\
\hline \multirow[t]{2}{*}{ Bank } & money & $98 \%$ \\
\hline & travellers cheques & $94 \%$ \\
\hline \multirow[t]{2}{*}{ Cleaners } & starched shirt & $99 \%$ \\
\hline & suit & $95 \%$ \\
\hline \multirow[t]{2}{*}{ Gas Station } & fuel & $99 \%$ \\
\hline & oil & $66 \%$ \\
\hline \multirow[t]{2}{*}{ Liquor Store } & beer & $86 \%$ \\
\hline & vodka & $100 \%$ \\
\hline \multirow[t]{2}{*}{ Hardware Store } & pliers & $95 \%$ \\
\hline & hammer & $98 \%$ \\
\hline
\end{tabular}

